\title{
Avoiding Post-Merger Corporate Downsize Restructuring: The Democratic Employee-Culture Fit Model (DeECFit)
}

\author{
Evangelos Markopoulos ${ }^{1,2}$, Ines Selma Kirane ${ }^{1}$, Emma Luisa Gann ${ }^{1}$, \\ Hannu Vanharanta ${ }^{3,4}$ \\ ${ }^{1}$ HULT International Business School, Hult House East, 35 Commercial Rd, E1 1LD, \\ London, United Kingdom \\ ${ }^{2}$ Turku University of Applied Sciences. Faculty of Engineering and Business \\ Joukahaisenkatu 3, ICT-City, C-wing, FI-20520 Turku, Finland \\ ${ }^{3}$ University of Vaasa, School of Technology and Innovations, Wolffintie 34, 65200 \\ Vaasa, Finland \\ ${ }^{4}$ Poznań University of Technology, Plac Marii Skłodowskiej-Curie 5, 60-965 \\ Poznań, Poland \\ evangelos.markopoulos@faculty.hult.edu,kinesselma01@gmail.com,emma.gann@hot- \\ mail.de, hannu@vanharanta.fi
}

\begin{abstract}
One major challenge in Mergers and Acquisitions is the best possible utilization of the human resources organizations offer. The judgment on employee redundancy is often highly controversial and risky. It can impact the motivation of the remaining employees, the corporate culture, and the reputation of the firm in the market. This paper addresses this challenge by introducing the Democratic Employee-Culture Fit Model (DeECFit) for Post-Merger Corporate Restructuring to avoid downsizing practices and maximize the utilization of the available human resources and intellectual capital. The model describes the relationship between the definition of the cultural values and the concentration of democratic values needed for an employee of either company to effectively join the Merger and operate under a symbiotic model by utilizing their human recourses. The democratic approach of the DeECFit Model provides the opportunity to all employers from both organizations to prove and justify their place in the Merger.
\end{abstract}

Keywords: Merger - Acquisition · Democracy $\cdot$ Downsizing $\cdot$ Rightsizing $\cdot$ Finance $\cdot$ Organization $\cdot$ Culture $\cdot$ Management $\cdot$ Human Recourses

\section{Introduction}

Mergers \& Acquisitions (M\&A) are common strategic tools for companies to acquire innovative products, capture new markets or simply scale on growth opportunities. The main reason for M\&A deals is synergies [1], for value creation. However, establishing effective synergies requires intensive efforts to successfully retain, realize and further create value through the utilization of the human resources. Value creation from 
synergies is expected and traded at the acquisition price and should be ensured from the post-merger integration onwards. Synergies rely on expected cash flows drawn by valuation models prior to and during the deal, but also on operations management, which is impacted by the corporate culture. Corporate culture relies on elements such as organization management, visions \& values, ethics, stories, common practices processes within the company's space and human intellectual capital. However, despite the critical role of the organizational culture in the merged organizations for value preservation and realization, the integration and human resources activities, are limited in an M\&A process approach. Such activities deal with understanding and comparing H\&R practices between the bidder and the target; assessing the culture of the target, guiding behaviors and attitudes of negotiators and due diligence teams, creating an integration transition structure, etc [1].

\section{Merger and Acquisition Challenges}

Mergers and Acquisitions can offer strategic advantages. However, the unique nature of an M\&A deal imposes risks realted to the business activities. Evidence shows that in most cases, organizations would have achieved better returns via bank account savings and investments, rather than acquisitions [2]. One popular reason for failures is wrong estimations of the M\&A potential. Less benefits from economies of scale and scope, lead to wrong revenue estimates which imply over-valuation of share prices that drop the stock price value [3]. Furthermore, human capital, resources and knowledge are often underestimated in the M\&A and the Post Merger Integration (PMI) processes. There is a taboo in engaging in human capital restructuring conversation, as this is often synonym of redundancies. According to McKinsey, $95 \%$ of the executives believe that cultural fit is "critical to the success of integration" and still, $25 \%$ describe the "lack of cultural cohesion and alignment" as the main reason for integration failure [4]. The absence of the proper understanding on the organizational culture results in demotivated employees or even withdrawal of the "key people" [2], reduces the loss of important knowledge and diminishes the synergies potential.

A renown example of a Merger failure attributed to cultural differences is the Daimler-Chrysler case. The two company cultures were at war from the very beginning due to different levels of formality, different expense philosophies and different work approaches, leading to huge financial losses and eventually many lay-offs primarily from Chrysler. With $60 \%$ of deals negative impacting shareholder value [3], clearly, it is not an easy tool to apply for strategic advantages. Despite those challenges leading to a failure rate of $83 \%$ on the long-term of M\&A deals, they are still on the rise with a worldwide increase of $8 \%$, according to the Institute of Mergers, Acquisitions and Alliances [5].

\section{Identifying, engaging and retaining Human Recourses}

Organizational culture is a key challenge corporate merges face on achieving the expected synergies during M\&A deals. Such synergies are not always effectives due to cultural discord. This paper attempts to provide a democratic approach to Corporate 
Cultural Fit in Post-Merger Integrations on achieving the best possible utilization of human resources whilst avoiding (deleterious)downsizing oriented restructuring. The model addresses the mindset required by decision-makers \& due-diligence teams on the topics of effective organizational restructure based on a cultural blend and human intellectual capital identification. This mostly entails on the identification and development of the human resources skills and abilities on their roles or the value expected to create in the merge, in order to avoid downsizing, meaning laying off employees who seem redundant.

Identifying and managing employee redundancy is one of the major challenges in the PMI (Post-Merger Integration) phase. Such a process comprises various levels of risk, embedded in each other, adding to the risk management and due diligence difficulty, leading to significant legal, and operations implications. It can impact the motivation of the remaining employees, the corporate culture, and the reputation of the firm to the market (press, customers, government). Examples of such risks can be found in historic merger failures such as the Daimler-Chrysler [6], Novell-WordPerfect [7] as well as the AOL-Time Warner Merger [8] and many others. Forbes estimated in 2015 that $83 \%$ of mergers fail for many reasons with the lack of forming common corporate culture to be a key one [9].

\section{Democratizing the Employee Culture Fit in Post Mergers and Acquisitions.}

The reduction of the excess human resources in M\&A impacts the employees who remain in organizations due to the fear of being in a similar situation sooner or later. Studies that tracked for nine years the performance of organizations that adopted downsizing strategies do not indicate outperformance against the ones who didn't [10]. Organizations with business environments that emphasize people-management practices, culture, and capabilities achieve competitive advantage [11]. Such practices emphasize on achieving the feeling of fairness among the employees. By applying fair processes, organizations reduce employee turnover, recruitment costs and legal cost, whilst generating knowledge that can support strategic initiatives, but also ignite an innovationbased culture [12].

To achieve organizational fairness especially in organizations formed from Mergers and Acquisitions, where organizational cultural differences significantly impact such a symbiosis, the concept of democracy on speech and actions can be applied. This research work presents the Democratic Employee-Culture Fit Model (DeECFit) for PostMerger Corporate Restructuring. The model is based on the Company Democracy Model [13], [14] and is structured through six evolutional operations levels. Those are evolved under three dimensions with each dimension to form a different organizational culture element all of which are needed to create the post-merger and acquisition organizational culture. To apply the DeECFit it is important to identify the concentration of democratic values needed for an employee of either company to effectively join the Merger. This is achieved by several challenges given to the employees on which they respond to demonstrate their value in the new organization while adopting the various cultural dimensions during the PMI phase. 
The model starts from the first level where the Merger challenges are introduced, and human resources skills and intellectual capital is identified and evolve all the way to level six where the challenges are resolved through the adaptation of the new organizational culture. The process provides two main outcomes to the merged organization. First, it identifies the employees willing and being able to join the merge, and second the knowledge generated from the challenge given. This knowledge can turn out to be the determinant factor and a base for either aligning the new organization's culture based on the human resources skills and intellectual capital or restructure the human resources to be aligned with the new organizational culture and strategy adaptation.

\section{The Democratic Employee-Culture Fit Model (DeECFit)}

Affective organizational cultures combine several dimensions in management and operations. The DeECFit Model is a culture driven classifying such dimensions under its Democratic Dimension, Value Chain Dimension and Culture Dimension (Fig. 1).

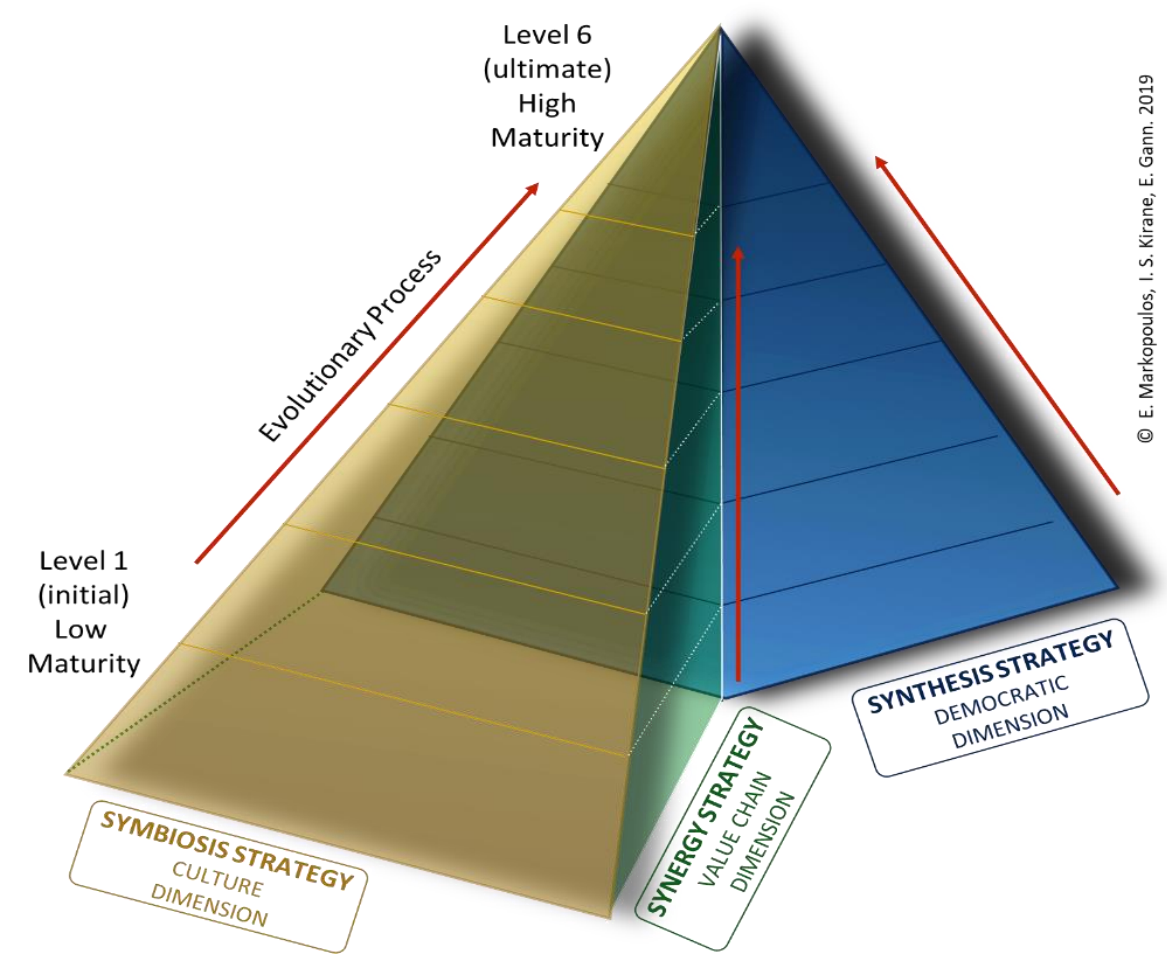

Fig. 1. Dimensions and Strategies of the Democratic Employee-Culture Fit Model

Each one of the culture dimensions is composed of six maturity levels forming a specific culture for each. The combination of these cultures forms the Post Merger/Acquisition Integration culture adopted by the employees of both organizations towards identifying their best place in it. 
The model provides the opportunity to all employees from both organizations to prove and justify their position in the merge while working under a common goal, forming the new post-merger organizational culture. The inner layers of the DeECFit Model form the operations strategies while the outer form the symbiosis strategy.

Specifically, the Democratic Dimension is based on the Company Democracy Model on building innovative competitive and globalized business operations. The model is driven by the values of democracy through which all the employees in the new (merged) organization can contribute to the success of the merge with their skills and intellectual capital. The Value Chain Dimension is based on value created through the democratic operations of the organization by increasing the organizational knowledge substantially and decreasing the dependability of the organization from external partners and suppliers. The Culture Dimension is based on the integration of the organizational culture each organization had prior to the merge, and the development of the new organization's culture through the operations that take place in the Value Chain and Democratic Dimensions. In other words, democracy ignites knowledge generation and utilization which creates an organizational value, which shapes the new post mergers organizational culture.

Each one of the DeECFit Model dimensions contributes to the development of the Post-Merger Integration organization strategy (Fig. 2).

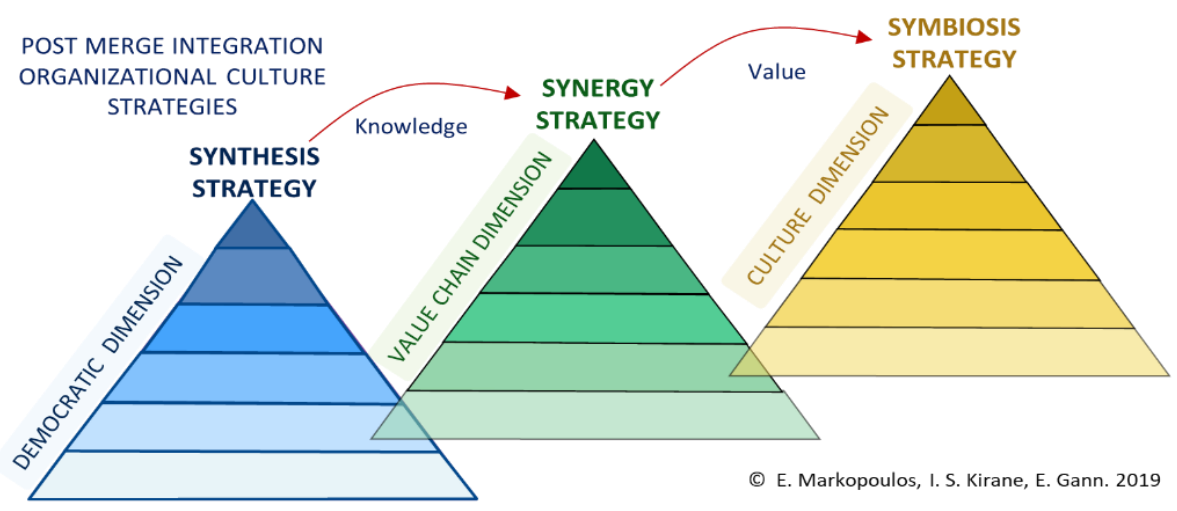

Fig. 2. DeECFit Model Dimension and Strategies Dependencies

The Democratic Dimension contributes towards a human resources synthesis strategy in which the employee's skills and intellectual capital is revealed through a career rewarding process. The Value Chain Dimension contributes towards a synergetic dimension between the human resources of the merged organizations to create a value chain process through complementarity and supplementary. Lastly, the Culture Dimensions contributes towards developing the Symbiosis Strategy by retaining characteristics from the organizational cultures of each merged organization and builds on them based on the evolution and effectiveness of the Synergy and Synthesis strategies. 


\section{The Democratic Dimension and the Synthesis Strategy}

The democratic dimension of DeECFit Model for the Post-Merger Integration organizational culture is composed of the six levels of the Company Democracy Model and its actual implementation process. (Fig. 3).

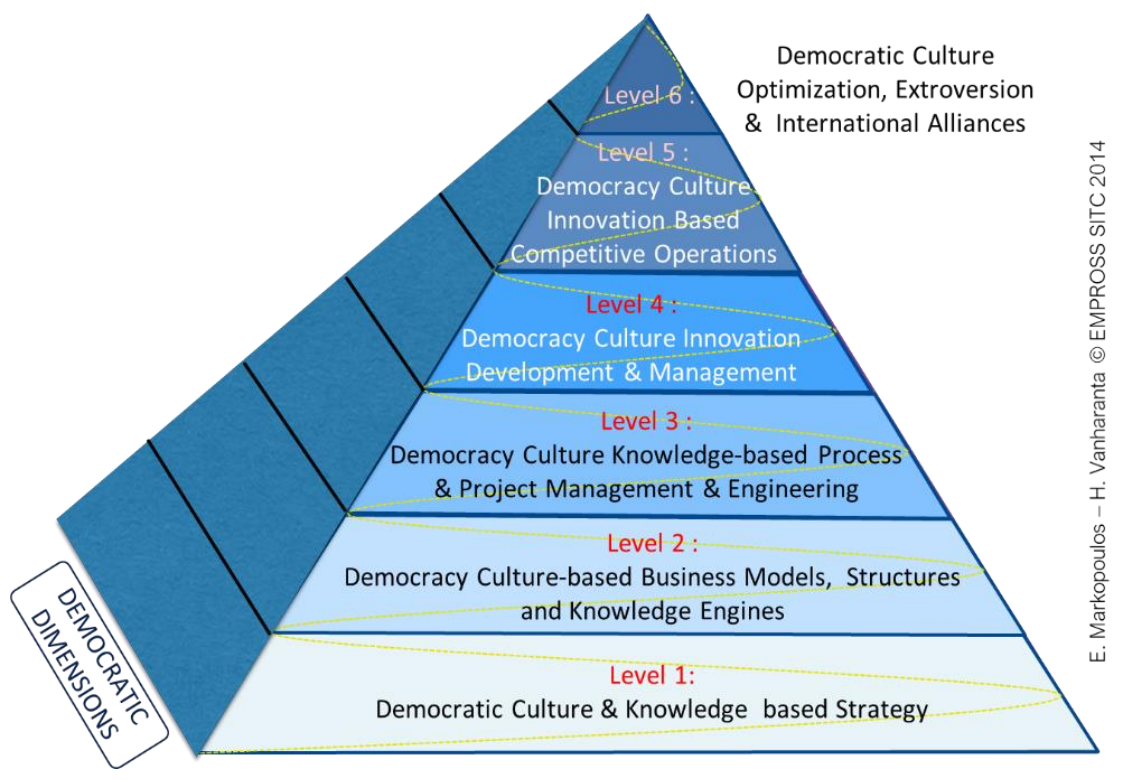

Fig. 3. The six levels of the Democratic Dimension in the DeECFit Model

The first level of this dimension provides a democratic culture where any employee from the merged organizations has the freedom and opportunity to share skills, knowledge, ideas, insights, and even visions and strategies. It is a level where fairness and equality are applied to all who feel that need to be treated fail and equal, seeking the chance and opportunity to demonstrate their value in the merged organization. The effectiveness of the first level impacts significantly the rest. The second level is based on the formation of teams around the ones who share knowledge in order to apply it on level three and turn it into innovation at level four. Level five identifies the competitive advantage gained from level four and with this advantage the organization can achieve international and global activities, partnerships and operations.

This Democratic Dimension implements the Post Merge Integration Synthesis Strategy as it blends the human resources form the two merged organizations under a democratic framework where they can equality collaborate, cooperate and co-evolve. It is a strategy that can, later on, determine the active employees from the non-active, the happy from the unhappy, and the engaged from the disengaged. With such a practical and fair employee evaluation the organizations can decide the degree of the downsizing strategy needed, and the ones that will be affected in the short and long run. 


\section{$7 \quad$ The Value Chain Dimension and the Synergy Strategy}

The Value Chain dimension of DeECFit Model for the post-merger integration organizational culture is composed of the six levels related to the Company Democracy Model and the Democratic Dimension. However, the goal, in this case, is the creation of a value chain that will be feeding the organization with the added value towards detaching itself from unnecessary and/or ineffective suppliers, partners and subcontractors. This is achieved by the effective utilization of the excess human recourses after the merger, to delivering value that was acquired from externals (Fig. 4).

The first level of this dimension emphasizes on identifying the needed value chain elements for the organization to successfully and effectively operate after the merge. This is achieved by mapping the expertise and knowledge acquired by the organization from externals (suppliers, subcontractors, etc), against the larger human resources pool the merged organizations now has. This mapping aims to direct the employee skills and intellectual capital towards delivering the needed work and service value that used to be outsourced. Based on the identification of those needs, level two creates the related teams, units and structures to work on them. Level three promotes co-opetitive, and not competitive management by supporting the teams of level two to work effectively and deliver the needed value. Level four builds on the value delivered by level three on turning it into competitiveness with creative operations, service innovation, patents, etc. Level five emphasizes on maintaining control of the value created, and keep on building on that from derivative elements, needs or enhancements. Lastly, level six commercializes globally the value created and measures it by its impact on the market, the economy and the society. At this level, the organization can export the added value created from the activities of this dimension.

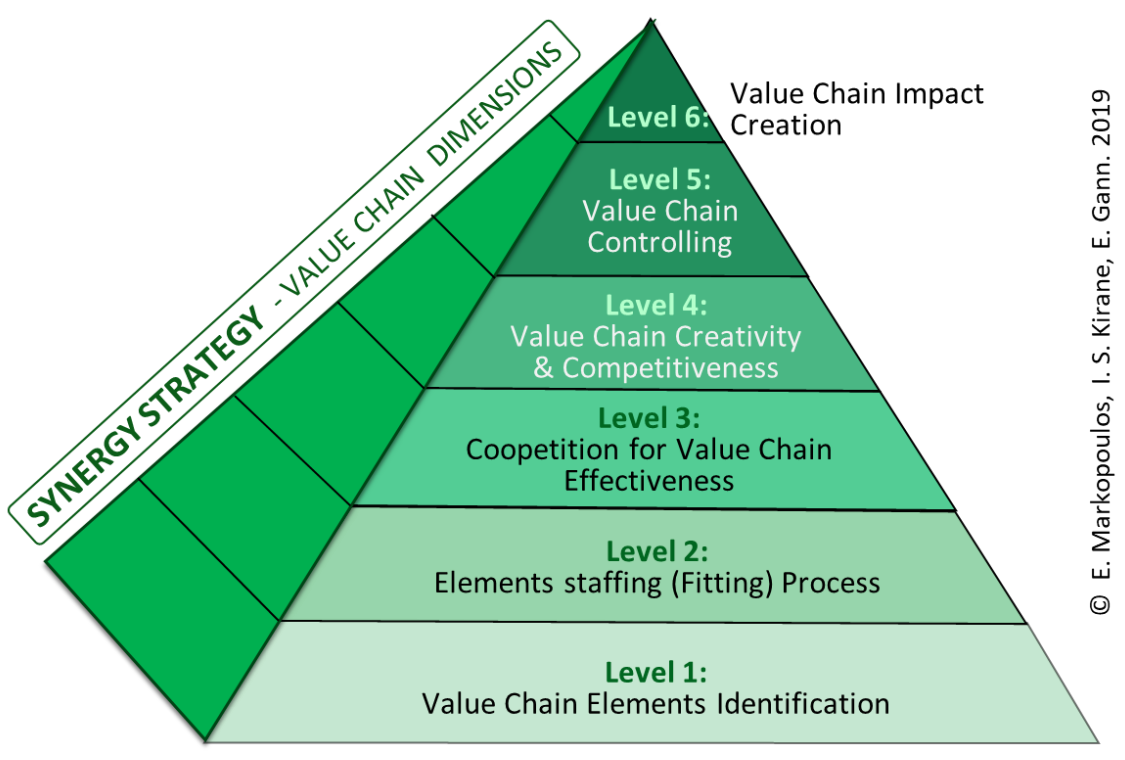

Fig. 4. The six levels of the Value Chain Dimension in the DeECFit Model 
This Value Chain dimension implements the Post Merger Integration Synergy Strategy as it synthesizes the new skills, expertise and intellectual capital of the new organizations to gain control over its operations by getting detached from its suppliers. The more value created by the new organization, the less will be spent on suppliers and external experts, and more autonomy and security the organization will have.

The Synergy Strategy differs from the Synthesis Strategy which is driven by the democratic dimension. The Synergy Strategy directs its employees on delivering specific needs, products or services the organizations obtained from externals. On the contrary, the Synthesis Strategy does not have specific requests from the employees but offers them the freedom to share and develop their knowledge and ideas best for the organization but not necessarily aligned with a specific goal such as the decrease of dependency from a supplier. A common denominator of the two strategies is that they both identify the performance and engagement of the employees to work together for the common good of the new organization. This performance impacts the need and the degree downsizing actions could have been taken otherwise.

\section{The Culture Dimension and the Symbiosis Strategy}

The Culture Dimension of DeECFit Model for the post-merger integration organizational culture is composed of six levels that act as an organizational culture umbrella over the Democratic and the Value Chain Dimensions. The goal of this dimension is to develop the new organizational culture by initially utilizing elements from the organizational cultures of the two merged organizations and enhanced them with the activities and work delivered in the Democratic and Value Chain Dimensions. (Fig.5).

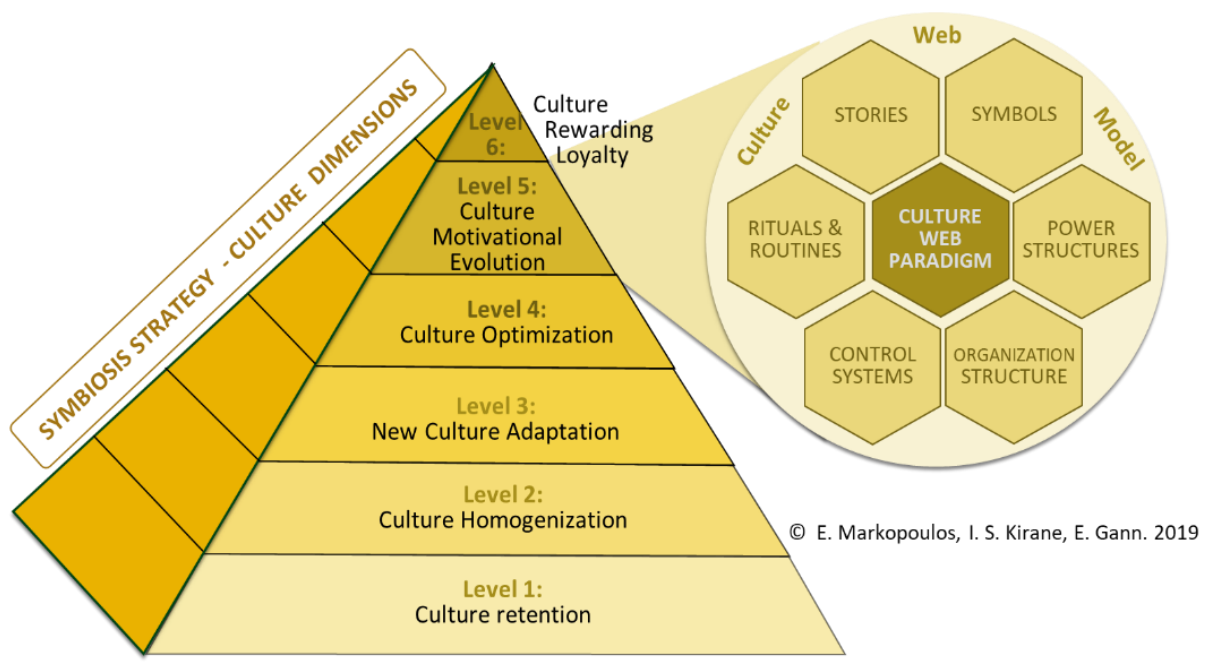

Fig. 5. The six levels of the Culture Dimension in the DeECFit Model

The first level of the Culture Dimension emphasizes on the retention of organizational cultural elements from the merged organizations. The more cultural elements 
from each organization are visible in the new organizational strategy the more engaged the employees will be. This is the most critical level of this dimension as the selection of the organizational culture elements that will be kept and integrated into the new organizational culture must be the ones the employees felt comfortable and proud to have around and work with. The second level emphasizes on the homogenization of the elements of level one towards integrating them with new organizational culture elements that did not existed in the pre-merged organizations but are critical for the new organizational culture. Such new elements can derive from the Democratic and Value Chain dimensions and can evolve in the organizational culture. The third level institutionalizes the new organizational culture as a standard practice, and level four optimizes the organizational culture by integrating best practices from the industry. Level five evolves the successful organizational experiences into innovative and motivational organizational culture elements though the culture web methodology [15] to extend the organizational culture beyond management and operational processes. Lastly level six aims on achieving employee, client and partner loyalty from the effective execution of the previous levels in conjunction with the effects of the Democratic and Value Chain dimensions. The degree of loyalty in a post-merger organization assures the degree of success from the reduction of employee, client and partner turnover.

This Culture Dimension implements the Post Merger Integration Symbiosis Strategy by creating the culture and the environment where all can work effectively, efficiently, fairly and proudly. The degree of success of the Symbiosis Strategy not only makes any downsizing decisions unnecessary but, on the contrary, increases the human resources of the post-merge organization with new roles needed to support, maintain and enhance the success achieved by the existing human resources.

\section{The Employee-Culture Fit Model for Post-Merger Corporate Restructuring}

The integration of the three dimensions and strategies compose the overall Democratic Employee-Culture Fit (DeECFit) Model visualized for consistency reasons in a sixlevel pyramid as well (Fig. 6). Each level integrates the activities that take place in the corresponding levels of the DeECFit Dimensions.

The first level of the model emphasizes on the identification of organizational knowledge from human resources. This knowledge can be expressed in the form of innovative ideas voluntarily and openly expressed by each employee or through employee engagement in value chain development challenges. The second level of the model emphasizes on goal-driven teaming and fitting initiatives. Teams are created around those who seek an opportunity or declare expertise to resolve value chain challenges. The third level emphasizes on merging organizational cultures for the development of the new one. This is impacted by the knowledge identified in level one and the role fitting of level two. The creation of an effective culture can empower the execution of the roles assigned, challenges given, and ideas proposed by the employees. The fourth level emphasizes on the value creation from the execution of the previous levels. This value can be tangible such as competitiveness, innovation, or intangible such as reputation or employee loyalty. The fifth level utilizes the value created in level four through new markers exploitation, internationalization or extroversion. Lastly, level 
six emphasizes on the impact the merge made in the local and global markets and societies. This impact determines the success of the merge and the number of employees that remain the new organization.

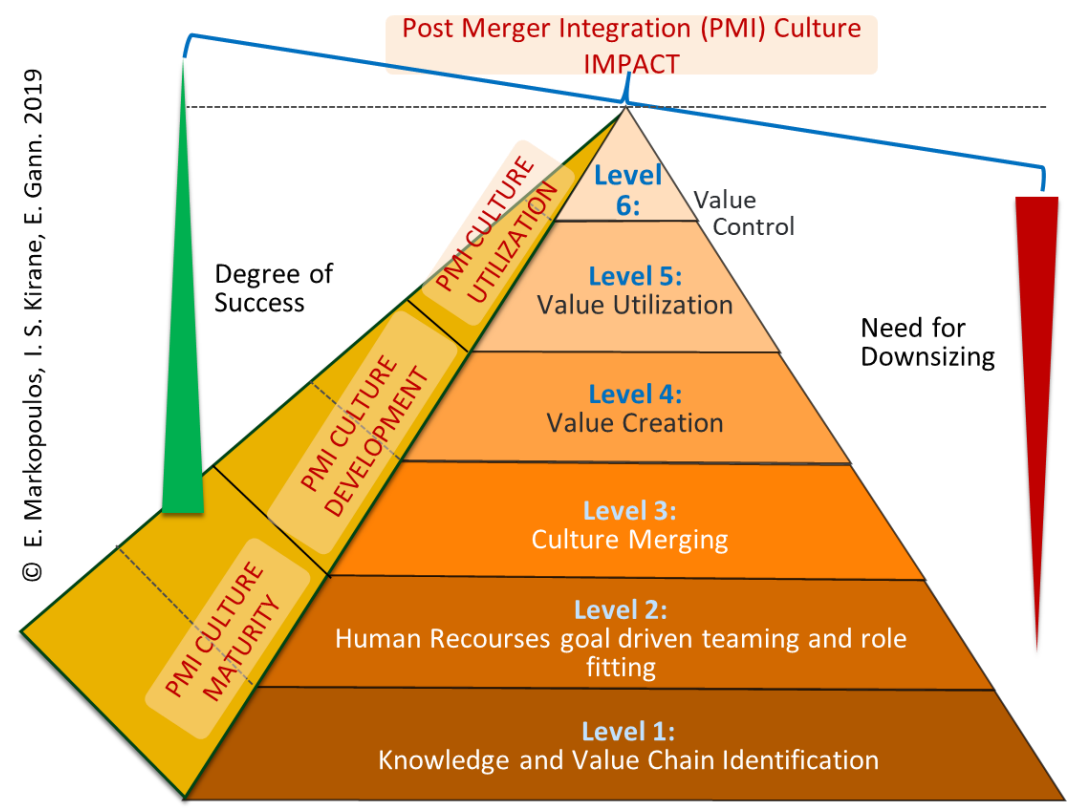

Fig. 6. The DeECFit Model: Key Structure.

The six levels of the model are categorized into three strategic goals. The first goal category is the maturity the merged organization shall achieve. It is the most challenging goal as this can determine the pace of success and the degree of downsizing. The second category is the development of the post-merger organization culture. This development requires the organizational maturity of the first goal to assure that the human recourses operate under a fair culture and fairly being placed in their roles in order to best perform operations and management. The last category is related to the utilization of the benefits gained from the previous categories. This will determine the impact of the Merger, its sustainability and the ability to reach the synergistic targets.

\section{Conclusions}

Mergers and acquisitions are often associated with the tragedy or job cuts, layoffs, due to downsizing programs implemented periodically or instantly in order to turn the new organization flexible, manageable and homogeneous.

However, a mind is a terrible thing to waste and human resources are actual minds that generate intellectual capital which cannot be wasted as it is never in excess. The DeECFit model has been developed to protect the people and their jobs while turning the merged organizations into success. The model is based on the principle that if one 
person can think then all people can think since they have all been created equally. However, some can think more technically, others more creatively and others more sentimentally. In modern organizations, all types of thinking are needed, especially in the ones created from mergers and acquisitions when knowledge plurality is one of the reasons for a Merger to happen. The DeECFit can be used to achieve rightsizing instead of downsizing, by offering equal opportunities for all employees to demonstrate their knowledge, skills, commitment to contribute and desire to stay in the organization

The three dimensions of the model, each one with a separate evolutionary methodology, and the strategies they form, contribute towards achieving rightsizing. The effectiveness of the execution of the DeECFit dimension reduces the need for downsizing and increase the need for more human resources to handle organizational success (Fig. 7).

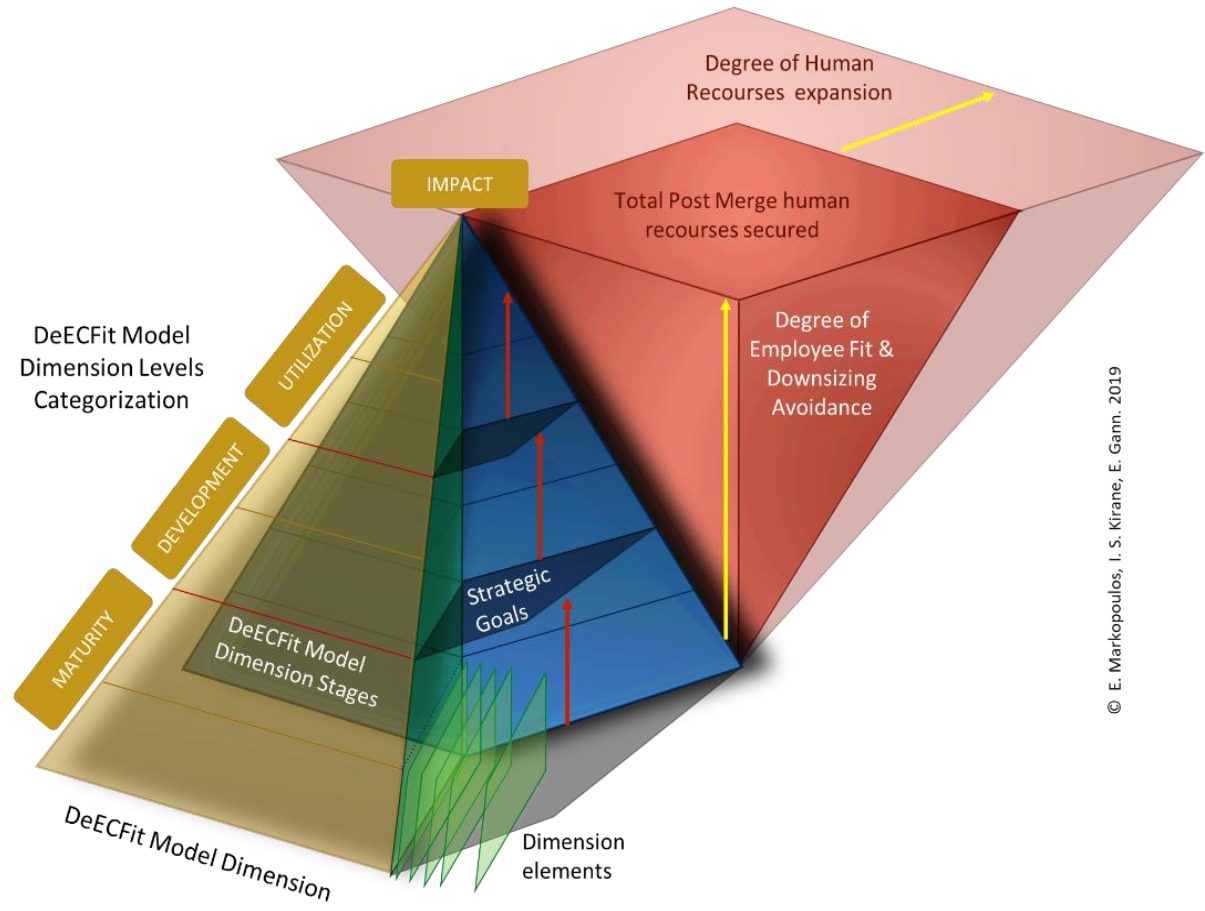

Fig. 7. The DeECFit Model Rightsizing Approach

The DeECFit Model creates jobs during the Integration period when the model is properly executed, meaning that the employees are properly engaged, indicated value and productivity. The lower levels of each dimension are the most complex to execute, therefore the need for downsizing and the risk for many people to lose their work is high. However, once each level is passed successfully this risk is being reduced significantly and can result not only in sustaining all jobs but also expanding to new jobs needed to sustain the effective operations of the newly merged organization. 


\section{References}

[1] Stahl G. K., Mendenhal M. E.: Mergers and Acquisitions: Managing Culture and Human Resources. Stanford Business Books. Stanford California (2005)

[2] Cartwright S.: Why mergers fail and how to prevent it. Business: The Ultimate Resource. QFinance. (2002).

[3] Walker C. : 3 Common M\&A Pitfalls, and How to Avoid Them. Harvard Business Review, Digital Article (2018)

[4] Engert O., Kaetzler B., Kordestani K., MacLean A.: Organizational culture in mergers: Addressing the unseen forces. McKinsey.com. https://www.mckinsey.com/business-functions/organization/our-insights/organizational-culture-in-mergers-addressing-the-unseenforces

[5] Zenner M., Berinstein B., Berkovitz T., Bansal A.: A shifting landscape for synergies. JPMorgan.(2019)

[6] Köhler HD.: From the Marriage in Heaven to the Divorce on Earth: the DaimlerChrysler Trajectory since the Merger. In: Freyssenet M. (eds) The Second Automobile Revolution. Palgrave Macmillan, London. (2009)

[7] Workhuman.: 6 Big Mergers That Were Killed by Culture (And How to Stop it from Killing Yours) https://www.workhuman.com/resources/globoforce-blog/6-big-mergers-that-werekilled-by-culture-and-how-to-stop-it-from-killing-yours

[8] Malone D., Turner J.: The Merger of AOL and TIME WARNER: A Case Study. Journal Of The International Academy For Case Studies. Vol. 16, Num.7. pp. 103--109

[9] Forbes. https://www.forbes.com/sites/georgebradt/2015/01/27/83-mergers-fail-leverage-a100-day-value-acceleration-plan-for-success-instead/

[10] SHRM: Employment Downsizing and its Alternatives. SHRM Foundation's Effective Practice Guidelines Series. (2009).

[11] Pfeffer, J.: The human equation: Building profits by putting people first. Boston: Harvard Business School Press. (1998).

[12] Brockner, J.: Why it's so hard to be fair. Harvard Business Review, 84(3), 122--129. (2006).

[13] Markopoulos E., Vanharanta H.: Democratic Culture Paradigm for Organizational Management and Leadership Strategies - The Company Democracy Model. Proceedings of the 5th International Conference on Applied Human Factors and Ergonomics AHFE 2014 (2014).

[14] Vanharanta H., Markopoulos E.: Creating a Dynamic Democratic Company Culture for Leadership, Innovation, and Competitiveness. 3rd Hellenic-Russian Forum. Sept.17. (2013).

[15] Johnson G., Whittington R., Scholes K.: Fundamentals of Strategy. FT Press; 2nd edition (2011) 